\title{
Pipe rheology of microfibrillated cellulose suspensions
}

\author{
Tuomas Turpeinen · Ari Jäsberg • Sanna Haavisto · Johanna Liukkonen • \\ Juha Salmela $\cdot$ Antti I. Koponen
}

Received: 11 July 2019/Accepted: 3 October 2019/Published online: 19 October 2019

(C) The Author(s) 2019

\begin{abstract}
The shear rheology of two mechanically manufactured microfibrillated cellulose (MFC) suspensions was studied in a consistency range of $0.2-2.0 \%$ with a pipe rheometer combined with ultrasound velocity profiling. The MFC suspensions behaved at all consistencies as shear thinning power law fluids. Despite their significantly different particle size, the viscous behavior of the suspensions was quantitatively similar. For both suspensions, the dependence of yield stress and the consistency index on consistency was a power law with an exponent of 2.4 , similar to some pulp suspensions. The dependence of flow index on consistency was also a power law, with an exponent of -0.36 . The slip flow was very strong for both MFCs and contributed up to $95 \%$ to the flow rate. When wall shear stress exceeded two times the yield stress, slip flow caused drag reduction with consistencies higher than $0.8 \%$. When inspecting the slip velocities of both suspensions as a function of wall shear stress scaled with the yield stress, a good data collapse was obtained. The observed similarities in the shear rheology of both the MFC suspensions and the similar behavior of some pulp fiber suspensions
\end{abstract}

T. Turpeinen · A. Jäsberg · A. I. Koponen ( $\square)$

VTT Technical Research Centre of Finland Ltd, P.O. Box 1603, 40401 Jyväskylä, Finland

e-mail: Antti.Koponen@vtt.fi

S. Haavisto · J. Liukkonen · J. Salmela

Spinnova Ltd, Jyväskylä, Finland suggests that the shear rheology of MFC suspensions might be more universal than has previously been realized.

Keywords Velocity profile - Ultrasound velocity profiling $\cdot$ Microfibrillated cellulose $\cdot$ Rheology $\cdot$ Yield stress $\cdot$ Viscosity $\cdot$ Shear thinning $\cdot$ Slip flow

\section{Introduction}

Micro/nanofibrillated cellulose (MNFC) is currently a material of high interest due to its sustainability and biodegradability, and its unique properties such as mechanical robustness, barrier properties, high specific surface area, lightness, and complex rheology. MNFCs can be isolated from wood or plant cell walls, and have lateral dimensions in the nanometer scale and length up to several micrometers. The fibrils may differ in physical properties depending on the production method and/or the raw material source. The MNFC suspensions produced using only mechanical treatment differ in size and morphology from MNFC suspensions produced using chemical, enzymatic, or carboxymethylation pretreatments followed by mechanical treatment (Desmaisons et al. 2017). Over the past decade, there has been an explosive growth in MNFC research, including improved MNFC production technologies, surface functionalization, 
characterization techniques, composites processing, self-assembly, optical properties, and barrier properties. The applications of MNFC are already numerous, varying now from a rheology modifier in cements, inks, drilling fluids and cosmetics, to a wide spectrum of products such as supercapacitors, transparentflexible electronics, batteries, barrier/separation membranes, and antimicrobial films (Klemm et al. 2011; Isogai 2013; Moon et al. 2016; Naderi 2017).

The rheological characteristics of various MNFC suspensions have become a widely discussed topic. Although knowledge of the rheological behavior is naturally important in the use of MNFC as a rheology modifier (Dimic-Misic et al. 2013a; Shao et al. 2015; Li et al. 2015) and stabilizer (Andresen and Stenius 2007; Winuprasith and Suphantharika 2013), such information is also needed for MNFC production (Pääkkönen et al. 2016; Delisée et al. 2010; Colson et al. 2016) and for other MNFC-related processes (Saarikoski et al. 2015; Hoeng et al. 2017; Shao et al. 2015; Kumar et al. 2017).

Due to the high aspect ratio of MNFC fibrils, their high surface area, and strong interfibrillar interactions, MNFC tends to form a gel even at low mass concentrations. MFCN gels can exhibit many complex phenomena, such as strong flocculation, yield stress, thixotropy, shear banding, complex, long-lasting transient flows, and shear thinning (Iotti et al. 2011; Saarikoski et al. 2012; Karppinen et al. 2012; Nechyporchuk et al. 2014; Martoia et al. 2015). Below the critical gelling concentration the behavior of the suspension can be close to Newtonian (Lowys et al. 2001; Lasseuguette et al. 2008).

There are many variables that can influence the rheological behavior of MNFC suspensions. These include consistency (Jowkarderis and van de Ven 2014, 2015; Charani et al. 2013), size distribution and morphology (Colson et al. 2016; Zhang et al. 2012; Saarikoski et al. 2012; Agoda-Tandjawa et al. 2010; Dimic-Misic et al. 2013b; Gourlay et al. 2018), composition (Pääkkönen et al. 2016), modifications to the cellulose surface (Samyn and Taheri 2016; Lasseuguette et al. 2008), ionic strength (Lowys et al. 2001; Saarikoski et al. 2012), and effects of various polymers (Karppinen et al. 2011; Agoda-Tandjawa et al. 2012; Naderi and Lindström 2014). Recent reviews on MNFC rheology can be found in Colson et al. (2016), Nechyporchuk et al. (2016) and Hubbe et al. (2017).
The viscous (shear-thinning) behavior of MFCN suspensions is usually well represented by the Herschel-Bulkley equation

$\tau=\tau_{y}+K \dot{\gamma}^{n}$.

Above, $\tau$ is shear stress, $\tau_{y}$ is yield stress, $\dot{\gamma}$ is shear rate, $K$ is consistency index, and $n$ is flow index. Yield stress is often omitted from Eq. (1), as it is typically small compared to the applied stress. One can then alternatively write

$\mu=K \dot{\gamma}^{n-1}$,

where $\mu$ is the shear viscosity of the suspension. The mechanism behind the shear-thinning behavior of MNFC suspensions, which is also seen with other fibrous materials (Derakhshandeh et al. 2011), is generally believed to be caused by adhesive interactions between the fibers. When shear rate increases, hydrodynamic shear forces are more effective in breaking the fiber-fiber contacts; this is reflected in a decreasing floc size and increasing orientation of the fibers. As a result, the efficiency of momentum transport in the suspension declines and the viscosity decreases (Iotti et al. 2011; Petrich et al. 2000; Bounoua et al. 2016). Note that the structural changes due to increasing shear forces are not always gradual; MNFC suspensions can also encounter abrupt structural changes, which are manifested by a sudden drop in viscosity (Lauri et al. 2017).

MNFC suspensions have a high flocculation tendency, and they often exhibit strong apparent wall slip due to wall depletion close to solid walls. The width of the wall depletion layer can vary between a few micrometers and a few hundred micrometers, depending on the MNFC grade, MNFC consistency, and flow conditions (Haavisto et al. 2015a; Lauri et al. 2017; Kataja et al. 2017). The slip flow makes it challenging to produce reliable information on shear rheology of MNFC using standard rotational rheometer geometries such as plate-plate, cone and plate, and cylinder cup geometries (Saarinen et al. 2014; Martoia et al. 2015; Vadodaria et al. 2018). This is specifically the case with small rheometer gaps and low shear rates, when the floc size may become comparable with the system size (Karppinen et al. 2012; Saarinen et al. 2014).

Figure 1 shows an example of two rheograms (viscosity-shear-rate curves) obtained with a cylinder 


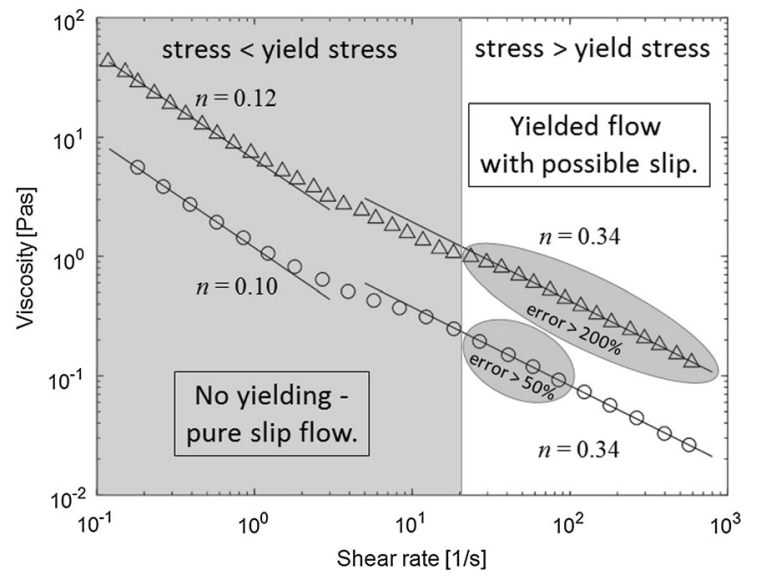

Fig. 1 Rheograms for a mechanically disintegrated MFC (for a light microscopy image of the MFC see Fig. 2b) for consistencies of $0.5 \%$ (spheres) and $1.0 \%$ (triangles) obtained with a cylinder cup rheometer with a $1 \mathrm{~mm}$ gap. Solid lines are fits of Eq. (2) to the measurement points (obtained fitting values of $n$ are also shown). There are only a couple of measurement points that reflect the true rheological behavior of the MFC suspensions with reasonable accuracy. Most points are below the yield stress, and above the yield stress errors in viscosity values are in most cases high. The data is from Saarinen et al. (2014) and Haavisto et al. (2015a)

cup rheometer for an a mechanically disintegrated MFC. We can see that even though the curves look reasonable, there are only a few legitimate measurement points that reflect the true viscous behavior of the suspensions with reasonable accuracy. When the shear rate is below $201 / \mathrm{s}$, shear stress is below the yield stress of the suspensions. The apparent flow is in this case only due to slip flow at the solid walls. Moreover, even above the yield stress, such slip flow generally introduces high errors to the measured viscosity. According to Vadodaria et al. (2018), scientific literature on the rheological properties of MNFC is replete with such erroneous viscosity data. Two shear thinning regions have been reported by many authors e.g. for MNFC suspensions under low and high shear conditions with a transition region between them. As we can see in Fig. 1, such curves do not necessarily reflect the real rheological behavior of the MNFC; the flow behavior may have been dominated by the slip flow and shearing may be (apparently) observed even below the yield stress. Unfortunately, yield stress values of MNFC are often unreported in the literature, making it difficult to assess which part of the rheogram is relevant for the analysis of the bulk viscosity. Values close to zero for exponent $n$ in Eq. (2) are, however, a clear warning sign. The shear stress is then approximately constant, which is a strong indication of slip taking place.

Thus, a challenge in MNFC rheology is to prevent the slip flow from influencing the data that are gathered. Rough walls can help, but do not necessarily eliminate this problem (Nechyporchuk et al. 2014). On the other hand, vane in cup geometry with a wide gap is known to decrease slip effects for many nonNewtonian fluids such as wood fiber suspensions (Mosse et al. 2012) and MNFC suspensions (Mohtaschemi et al. 2014a). Schenker et al. (2018) studied shear viscosity of mechanically disintegrated MFC in the consistency range of $0.5-2 \%$. According to their observations, the effect of slip was strong in a cylinder cup geometry with smooth walls, and rough walls decreased the slip only slightly. However, a vane in cup geometry with a wide gap and rough walls was found to give more reliable results. The difference between the measured viscosities was almost a factor four at the shear rate of $100 \mathrm{1} / \mathrm{s}$. Note that a wide gap is not always problem-free. It may introduce heterogeneous flow in the vane geometry, which must be properly addressed in order to obtain correct results. A wide gap may also cause the system to be susceptible to secondary flows (Mohtaschemi et al. 2014a).

An alternative method for measuring shear rheology is to use a pipe with a diameter much higher than a typical structural element (fiber, fibril or floc) of the suspension. With this setup, it is possible to obtain information on the flow behavior of MNFC suspensions in realistic process-like conditions, as the suspension behaves inside the pipe like a continuum medium and many artefacts seen in traditional rotational rheometers are eliminated. However, the slip flow may also distort the results here, as slip may increase the total pipe flow rate of MNFC suspensions considerably (Haavisto et al. 2015a; Nazari et al. 2016; Lauri et al. 2017).

An attractive option to eliminate the effect of slip in the rheological analysis is to use velocity profiling, i.e. to measure the flow profile of the MNFC suspension in the research geometry explicitly (Salmela et al. 2013; Haavisto et al. 2015a; Lauri et al. 2017; Haavisto et al. 2017; Kataja et al. 2017). When combined with pressure loss (e.g. capillary/pipe flow) or torque (e.g. rotational rheometers) measurements, this data can be used to characterize the rheological properties of the suspension. Although the use of magnetic resonance 
imaging (MRI) and optical coherence tomography (OCT) is increasing (Haavisto et al. 2017), ultrasound velocity profiling (UVP) is the most popular (noninvasive) flow measurement technique used in velocity profiling. It facilitates velocity measurements at relatively low cost and sets minimal requirements for the measurement setup (Powell 2008; Haavisto et al. 2011).

In this paper, we analyse and compare the shear rheology of two MFC suspensions (see Fig. 2) in the consistency ranges of $0.2-1.5 \%$ and $0.5-2.0 \%$. The particle size of these two materials was estimated to differ by an order of magnitude. We used a vertical pipe rheometer combined with ultrasound velocity profiling, and a horizontal pipe rheometer combined with OCT velocity profiling. Some of the presented data has been published earlier in two conference papers (Haavisto et al. 2011; Salmela et al. 2013). In this paper we combine this data with some novel data and go much deeper in our analysis. In addition to yield stress and shear viscosity, we study the slip behavior of the MFC suspensions, as this topic has hitherto been mostly neglected in the existing literature.

\section{Materials and methods}

\section{Microfibrillated cellulose}

Two microfibrillated celluloses were used in this study. The first one (MFC1) was a commercial product Celish KY-100G (Daicel Chemical Industries, Japan), made mechanically from purified softwood pulp (see Fig. 2a). The size distribution of these fibrils is very wide, ranging from microscale to nanoscale. According to Tatsumi et al. (2002) the average width and length of the Celish fibers are $15 \mu \mathrm{m}$ and $350 \mu \mathrm{m}$, respectively. The finest $20 \%$ mass fraction, however, has the average width of $50 \mathrm{~nm}$ and the average length of $8 \mu \mathrm{m}$ (Varanasi et al. 2013). The surfaces of Celish fibrils are very strongly fibrillated. For this study, seven measurement sets of MFC were prepared, with mass consistencies varying from 0.17 to $1.53 \%$. According to Raj et al. (2016) the gel point of the suspension is $0.24 \%$ and surface charge is $43 \mu \mathrm{eq} / \mathrm{g}$. With the lowest consistencies $0.17 \%$ and $0.28 \%$ the gel was indeed quite weak, and the qualitative behavior of the suspensions was liquid-like.
The second microfibrillated cellulose (MFC2) was prepared from never-dried bleached Kraft birch pulp by grinding three times in a supermasscolloider (Masuko Sangyo, Japan). Prior to grinding, the pulp was changed to its sodium form and washed with deionized water to obtain an electrical conductivity less than $10 \mathrm{~S} / \mathrm{cm}$, according to a procedure introduced by Swerin et al. (1990). The dry matter content after grinding was $2 \%$. As we see in Fig. $2 b$, while there are still some long fibers present in MFC2, the fibril size of MFC2 is on the average clearly smaller than for MFC1. This is reflected also in the wall depletion layer of MFC2 which is an order of magnitude thinner than for MFC1 (Salmela et al. 2013; Lauri et al. 2017). The gel point and surface charge of this type of MFC made with 5 homogenization passes are $51 \mu \mathrm{eq} / \mathrm{g}$ and $0.1 \%$, respectively (Raj et al. 2016). For MFC2, which had 3 homogenization passes, surface charge is slightly smaller, 45-50 $\mu \mathrm{eq} / \mathrm{g}$. The gel point, on the other hand, is slightly higher, $0.2-0.3 \%$. The surface charges of MFC1 and MFC2 are thus very similar and charge should have only minor effect when comparing their rheology. For the rheological experiments, MFC2 samples were diluted with deionized water to consistencies of $0.5 \%, 1.0 \%$ and $2.0 \%$.

\section{Ultrasound velocity profiling}

Ultrasound velocity profiling (UVP) is a well-established experimental technique in applications of fluid dynamics and engineering involving flow measurements (Takeda 2012). It is based on using an emitterreceiver probe to send a series of short ultrasound bursts into the flow, and detecting the echoes issuing from the target particles moving along with the flow. The spatial location of the particles is acquired with the time-of-flight method using the known velocity of sound in the flowing medium. The determination of fluid velocity is based on the estimation of the mean phase shift of consecutive echoes originating from the defined depth.

\section{Optical coherence tomography}

Optical coherence tomography (OCT) is a light-based imaging method, which enables non-contact, micronscale spatial resolution measurement of scattering opaque materials (Drexler and Fujimoto 2008). OCT uses interference of a low coherence light to record 
depth-dependent reflectivity and velocity profile. A standard OCT setup includes a low-time coherence light source, such as a superluminescent diode and a Michelson interferometer. Depending on the OCT technology, axial scanning rates can vary over a range of tens to hundreds of $\mathrm{kHz}$. The actual imaging depth depends significantly on the optical properties of the material and can vary from micrometers to a few millimeters.

\section{Pipe rheometers}

The vertical pipe rheometer consisted of two chambers connected by a smooth acrylic pipe with an inner diameter of $16 \mathrm{~mm}$. Velocity profiles of the flow across the pipe were obtained with UVP together with pressure difference and mass flow measurements. The flow in the measurement pipe was driven by gravity and by optional overpressure in the upper chamber. The flow rate was controlled by a valve in case of pure gravity-driven flow or by a pressure regulator when the overpressure was used. The pressure difference in the measurement pipe was measured over a distance of $0.90 \mathrm{~m}$, and the first pressure measurement point was located $300 \mathrm{~mm}$ from the pipe inlet. A DOP2000 device equipped with an $8 \mathrm{MHz}$ ultrasound probe was used for the UVP measurements. The position of the UVP measurement was located 77 pipe diameters from the pipe inlet. For more details on the measurement setup see Haavisto et al. (2011). The pipe rheometer data was used for the analysis of yield stress, viscosity and slip flow of the MFC suspensions.

The horizontal pipe rheometer consisted of a chamber connected with a hose to a $1500 \mathrm{~mm}$ long optical grade glass pipe with an inner diameter of $8.6 \mathrm{~mm}$. The flow was driven by gravity and pressurized air. The pressure difference in the measurement pipe was measured over a distance of $1.0 \mathrm{~m}$, and the first pressure measurement point was located $450 \mathrm{~mm}$ from the pipe inlet. The OCT measurement was located 113 diameters from the inlet. A Telesto Spectral Domain OCT instrument was used for the OCT measurements. For more details on the measurement setup see Salmela et al. (2013). The pipe rheometer data was used to obtain extra insight for the wall slip behavior of MFC1.
Determination of yield stress from the pipe rheometer data

The advantages of a pipe rheometer in the determination of yield stress are the size of the flow geometry and flow conditions that are relevant for real-life processes. We present two options for the yield stress analysis using a pipe rheometer.

The yield stress can be approximated by the value of the total shear stress at the boundary of the fiber plug as $\tau_{y}=\tau_{w}\left(1-R_{0} / R\right)$, where $R_{0}$ is the distance from the pipe wall at which the fiber plug breaks. The edge of the fiber plug was determined directly from the velocity profiles using their first derivative. While representing the true shear rate of the flow in the pipe rheometer, the first derivative becomes zero at the position where the shear stress reaches the yield stress value. However, as the measurement time is finite, there are always variations in the shear rate caused by both UVP noise and consistency fluctuations. The location of the fiber plug edge was determined by fitting straight lines to the velocity profile half. The number of data points included in the fit was decreased starting from the wall. The removal of the profile data points in the sheared layer resulted in a decreasing fit error, which saturated when the plug region was reached. The yield stress was approximated from such a point of saturation.

In the second approach for determining the yield stress, the fluctuations of the shear rate were utilized. The magnitude of the fluctuations was approximately constant below the yield stress, whereas above the yield stress the fluctuations increased rapidly with increasing shear stress. To calculate the yield stress, the shear rates from all velocity profiles were binned such that all the shear rates related to the same shear stress region were in the same bin. Next, the standard deviation of the shear rate for each bin was calculated and the data was plotted on a loglog-scale. The yield stress was obtained from the cross point of two power law curves (straight lines on the loglog-scale) that were fitted to the data. We should emphasize that this approach is purely heuristic, and we do not currently have any theoretical justification for it. It worked rather well for the MFC suspensions used in this study, but more measurement data is needed before final conclusions can be drawn. 

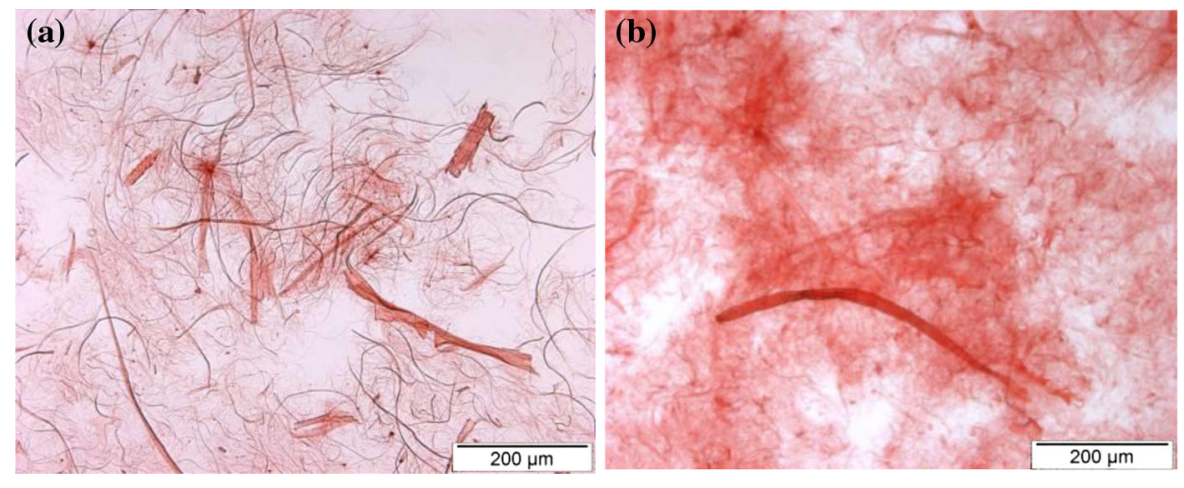

Fig. 2 Light microscopy images of a MFC1, and b MFC2. (Kinnunen-Raudaskoski et al. 2013)

Determination of viscosity and slip velocity from the pipe rheometer data

Before analysis, a procedure of normalization was applied to the UVP data in order to eliminate the systematic uncertainties related to the absolute velocity values in the measured velocities, e.g. due to the inclination angle between the ultrasound beam and the direction of flow. The time-averaged velocity profiles were normalized with the independently measured flow rate as

$v_{\text {norm }}=\frac{Q_{\mathrm{REF}}}{Q_{\mathrm{UVP}}} v_{\mathrm{UVP}}$

where $Q_{\mathrm{REF}}$ is the measured flow rate and $Q_{\mathrm{UVP}}$ is the flow rate integrated using the UVP profile. The measured and normalized UVP velocity profiles were then used to calculate the fluid viscosity locally by

$\mu(r)=\frac{\tau(r)}{\dot{\gamma}(r)}$.

Here, $\dot{\gamma}(r)$ is the local shear rate

$\dot{\gamma}(r)=\frac{d v(r)}{d r}$,

which is obtained directly from the measured velocity profile $v(r)$, and

$\tau(r)=\tau_{w} \frac{r}{R}$

is the local shear stress at a distance $r$ from the center of the pipe having a radius $R$. Above, $\tau_{w}$ is the wall shear stress

$\tau_{w}=\frac{R}{2} \nabla P$, where $\nabla P$ is the pressure gradient. Each measured velocity profile thus gives viscosity values in the range of shear rates (and shear stresses) present in the measured velocity profile.

The power law behavior of MFC suspensions also makes it possible to calculate the suspension viscosity by fitting to the UVP data the analytical power law velocity profile (for simplicity we neglect yield stress, $\left.\tau_{y}\right)$

$v(r)=v_{s}+\frac{n}{n+1}\left(\frac{\nabla P}{2 K}\right)^{\frac{1}{n}}\left[R^{\frac{n+1}{n}}-r^{\frac{n+1}{n}}\right]$.

Above, $n, K$, and $v_{s}$ (slip velocity) are fitting parameters. In the fitting, we assumed the parameters $n$ and $K$ to be constant for all profiles with the same consistency, and the profile fitting was performed in two phases. First, the individual profiles were fitted to each measured profile, and the original profile data was scaled with the ratio of mean velocity from the mass flow to the fitted profile integral. Then the profiles of the same consistency were fitted simultaneously, keeping the parameters $n$ and $K$ the same for all fitted profiles, whereas $v_{s}$ was unique for each profile. Figure 3 shows an example of such a fit.

Based on our earlier experience (Haavisto et al. 2015b), the OCT velocity profiles were assumed to be sufficiently accurate as such. The measured OCT velocity profiles (see Fig. 4) were fitted by the empirical formula (Salmel et al. 2013)

$v(y)=\dot{\gamma}_{w}^{a} y+v_{s}\left(1-e^{-\frac{y}{\lambda_{w}}}\right)$,

where $y$ is distance from the wall and $\dot{\gamma}_{w}^{a}, v_{s}$, and $\lambda_{w}$ are free parameters (see Fig. 4). Parameter $\dot{\gamma}_{w}^{a}$ is the apparent shear rate at wall, $v_{s}$ is (apparent) slip 


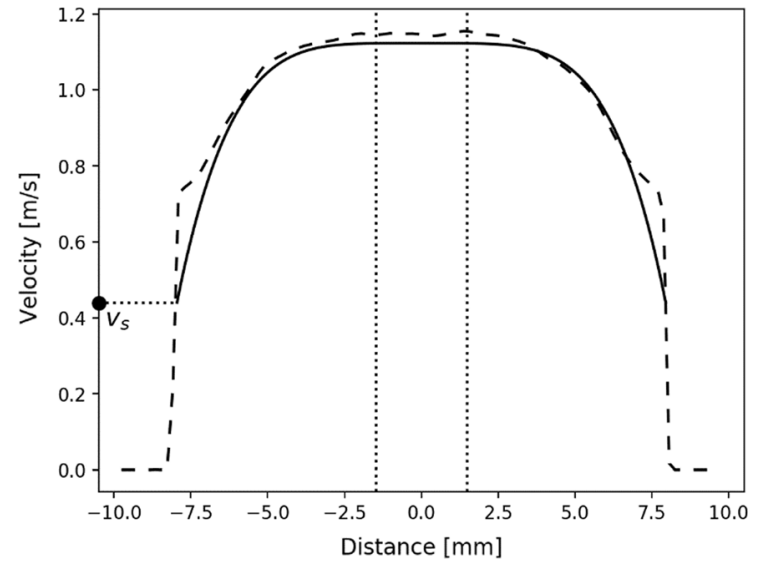

Fig. 3 A measured UVP velocity profile for MFC1 (dashed line), velocity profile given by the fitted model (solid line), and the radial positions where the shear stress equals yield stress (vertical dotted lines). The fitted slip velocity $v_{s}$ is also shown. The S-shaped distortions seen close to the walls are caused by the averaging effects across the finite measurement sample volume in the flowing fluid. (Kotze et al. 2013)

velocity, and $\lambda_{w}$ is the characteristic thickness of the apparent slip layer. We can see in Fig. 4 that in a narrow near-wall region, the velocity profile is very steep and rapidly approaches zero with decreasing distance from the wall with no actual wall slip. The (apparent) wall slip is due to velocity deficit in a narrow slip layer where the consistency is smaller than the bulk consistency.

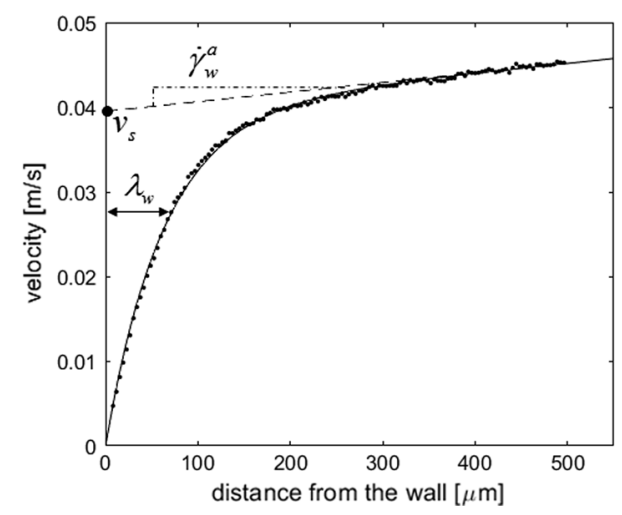

Fig. 4 Example of a fitted velocity profile Eq. (9) to a measured OCT velocity profile for MFC1. Also shown are graphical interpretations of the three free fitting parameters $\dot{\gamma}_{w}^{a}$, $v_{s}$ and $\lambda_{w}$

\section{Results}

Pressure loss

Figure 5 shows the measured pressure loss as a function of mean velocity for MFC1 and MFC2. As a comparison, the theoretical curve for water is also shown. The pressure drop curves resemble typical behavior of pseudoplastic (shear thinning) fluids. For MFC1, pressure loss temporarily levels or even drops for consistencies of $0.8-1.7 \%$ in both rheometer geometries, when the mean velocity exceeds $0.06 \mathrm{~m} /$ $\mathrm{s}$. This phenomenon, drag reduction, is caused by wall slip; similar behavior is also observed with other fiber suspensions, such as pine pulp (Jäsberg 2007). The effect appears to be stronger in the vertical pipe. The pressure loss of MFC1 has more variation in Fig. 5b when compared to Fig. 5a, especially when mean velocity exceeds $0.05 \mathrm{~m} / \mathrm{s}$. This is probably related to the dynamics of the slip boundary layer.

Yield stress

For MFC1, yield stress was determined from the first derivative of the velocity profile (see Fig. 6a). This approach did not work so well for MFC2, as no clear threshold could be found between fluctuating and nonfluctuating regions. For this reason, the yield stress of MFC2 was determined from the fluctuations of shear rate (see Fig. 6b). Figure 7 shows the measured yield stresses as a function of consistency. We can see that there is a good agreement between the pipe rheometer data and corresponding values found from the literature. Notice that in Varanasi et al. (2013) Celish was first filtered through two fabric filters with $100 \mu \mathrm{m}$ openings and then the filtrate was centrifuged. After centrifuging, the supernatant was discarded and only the MFC fibrils at the bottom were collected. The yield of this process was $20 \%$. It is interesting that despite the significantly different size distributions the original Celish, MFC1, measured in this work, and its $20 \%$ fines fraction, measured by Varanasi et al. (2013), have practically identical yield stresses.

Varanasi et al. (2013) and Saarinen et al. (2014) used vane in cup (17 mm gap) and cylinder cup (1 mm gap) geometries for MFC1 and MFC2, respectively. A $1 \mathrm{~mm}$ gap in the vane in cup geometry, on the other hand, resulted in twofold higher yield stress for MFC1 (Haavisto et al. 2011). It appears that MFC1 was too 

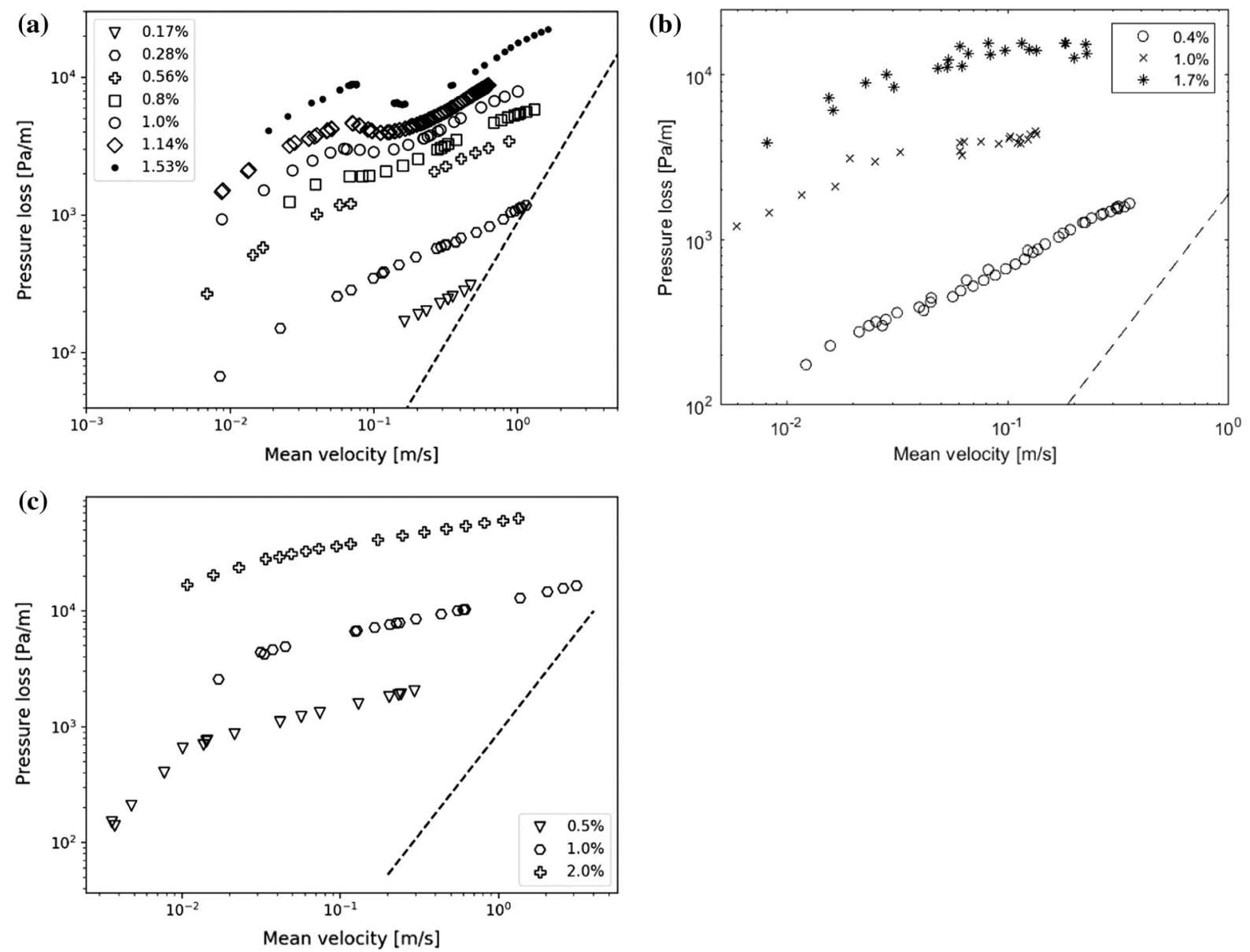

Fig. 5 Mean velocity versus pressure loss for a MFC1 in the vertical pipe, $\mathbf{b}$ MFC1 in the horizontal pipe (the variations are probably due to the sensitivity of the slip velocity in this

coarse for the small gap of this geometry. Note that in Tatsumi et al. (2002), a plate-plate geometry $(1.5 \mathrm{~mm}$ gap) was used with an MFC grade similar to MFC1. The obtained yield stress values were more than an order of magnitude smaller than in this work, probably due to severe slip flow on the plate walls.

For pulp fiber cellulose suspensions, the yield stress typically correlates with the consistency of the suspension such that

$\tau_{y}=a c^{b}$.

According to Dalpke and Kerekes (2005), exponent $b$ slowly approaches the value of ca. 2.4 when the fiber length (or aspect ratio) increases. It is interesting that the exponent $b$ is here very close to this value for both MFC suspensions (see the power law fits in Fig. 7). In

geometry) and c MFC2 in the vertical pipe. The dashed line shows the pressure loss for water

Nazari et al. (2016), the exponent was 3.2 in the consistency range of $2-9 \%$ for a mechanically made MFC. In their study five different methods for measuring the yield stress were analyzed and it was found that the results could deviate by as much as a factor of 4. In Tatsumi et al. (2002), the exponent was 2.0 for five different cellulose micro- and nanofibrils originating from wood, cotton and bacterial cellulose. However, as discussed above, these results may have suffered from severe slip in the rheometer geometry.

\section{Shear viscosity}

Figure 8 shows the viscosity of MFC1 and MFC2 as a function of shear rate determined with a local derivative Eq. (4). The solid lines show power law fits, Eq. (2), to the viscosity data. The variation in viscosity 
(a)

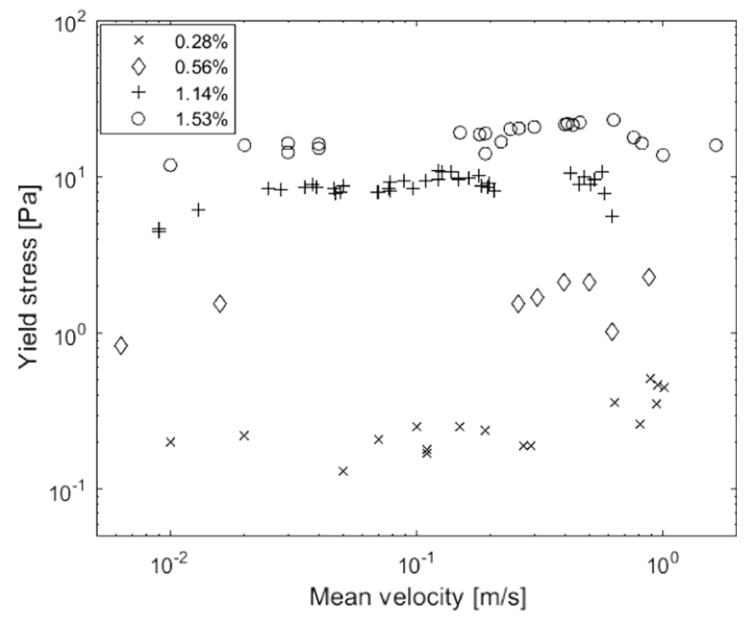

(b)

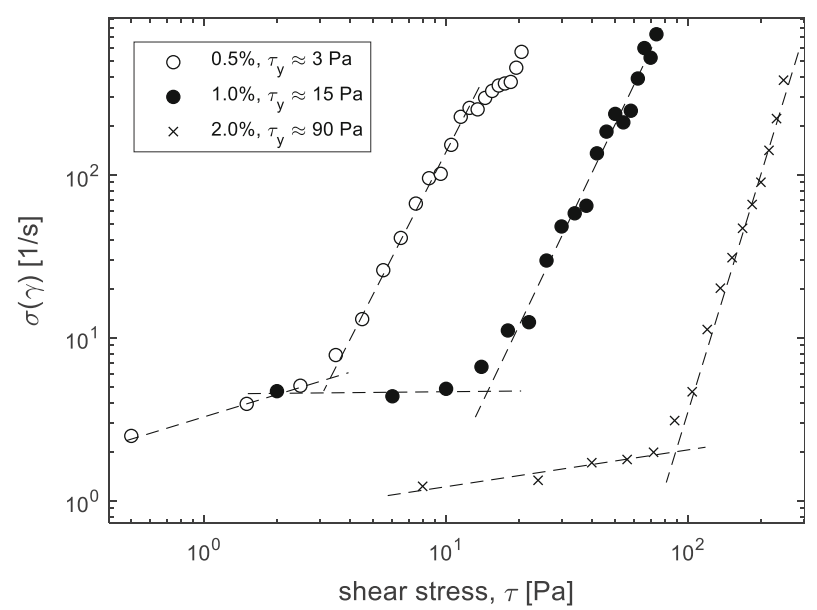

Fig. 6 a Examples of yield stress values calculated for MFC1 at the plug boundary for different flow rates. b Estimating the yield stress of MFC2 from shear rate fluctuations

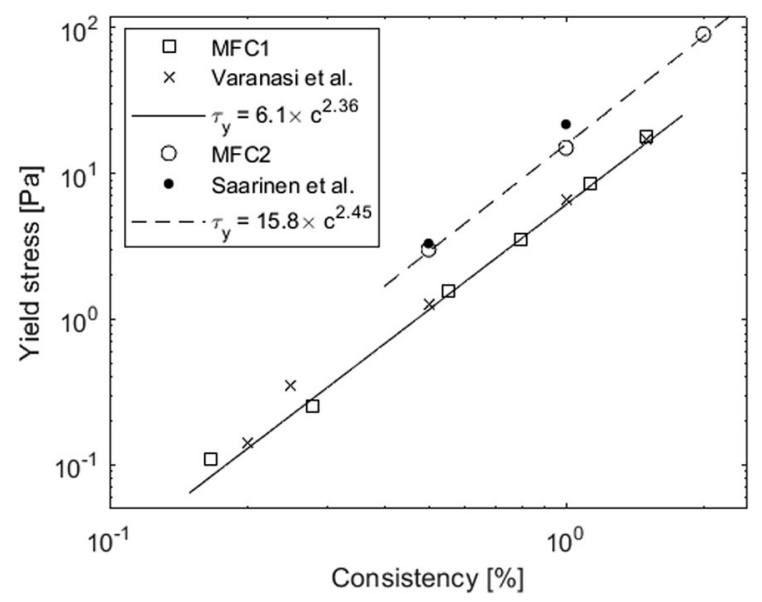

Fig. 7 Yield stress as a function of consistency for the MFC suspensions. As a comparison, results obtained for a fine fraction of MFC1 with a vane geometry (Varanasi et al. 2013) and for MFC2 with a cylinder cup geometry (Saarinen et al. 2014) are also shown. The power law fits have been applied in our data

values is of the order of a factor of two, but due to the high number of data points, the power law fits should be quite accurate. Table 1 shows the obtained power law coefficients $K$ and $n$. As a comparison, the coefficients obtained by fitting the velocity profile, Eq. (8), are also shown. Although there is some discrepancy in the results with the two lowest consistencies, profile fitting appears to be a quite viable alternative for determining viscosity for the used measurement setup. For further analysis, we use below the parameters obtained with a local derivative.

Figure 9a shows the flow index $n$ as a function of consistency for both MFC suspensions. A power law fit for flow index $n$ gives

$n=0.27 \times c^{-0.36}$.

Note that similar scaling behavior of $n$ has been observed e.g. for another mechanically made MFC (Schenker et al. 2018) and for an MFC made with TEMPO-oxidization (Mohtaschemi et al. 2014b).

Figure $9 \mathrm{~b}$ shows the consistency index $K$ as a function of consistency for both MFC suspensions. Many studies have shown that the dependence of MNFC viscosity on consistency follows a power law. Here we get for MFC1

$K=7.2 \times c^{2.37}$.

We could find only one paper in which the dependence of consistency index on consistency has been reported explicitly. Schenker et al. (2018) studied mechanically made MFC in the consistency range of $0.5-2.0 \%$. They obtained 2.8 for the exponent in Eq. (12). In some papers the consistency indices have been reported for several consistencies and the exponent in Eq. (12) can be calculated. Lasseuguette et al. (2008) and Mohtaschemi et al. (2014a) studied TEMPO-oxidized NFC. For consistency ranges of $0.1-0.5 \%$ (Lasseuguette) and $0.25-1.0 \%$ (Mohtaschemi), the exponents were 2.7 and 2.4 , respectively. 

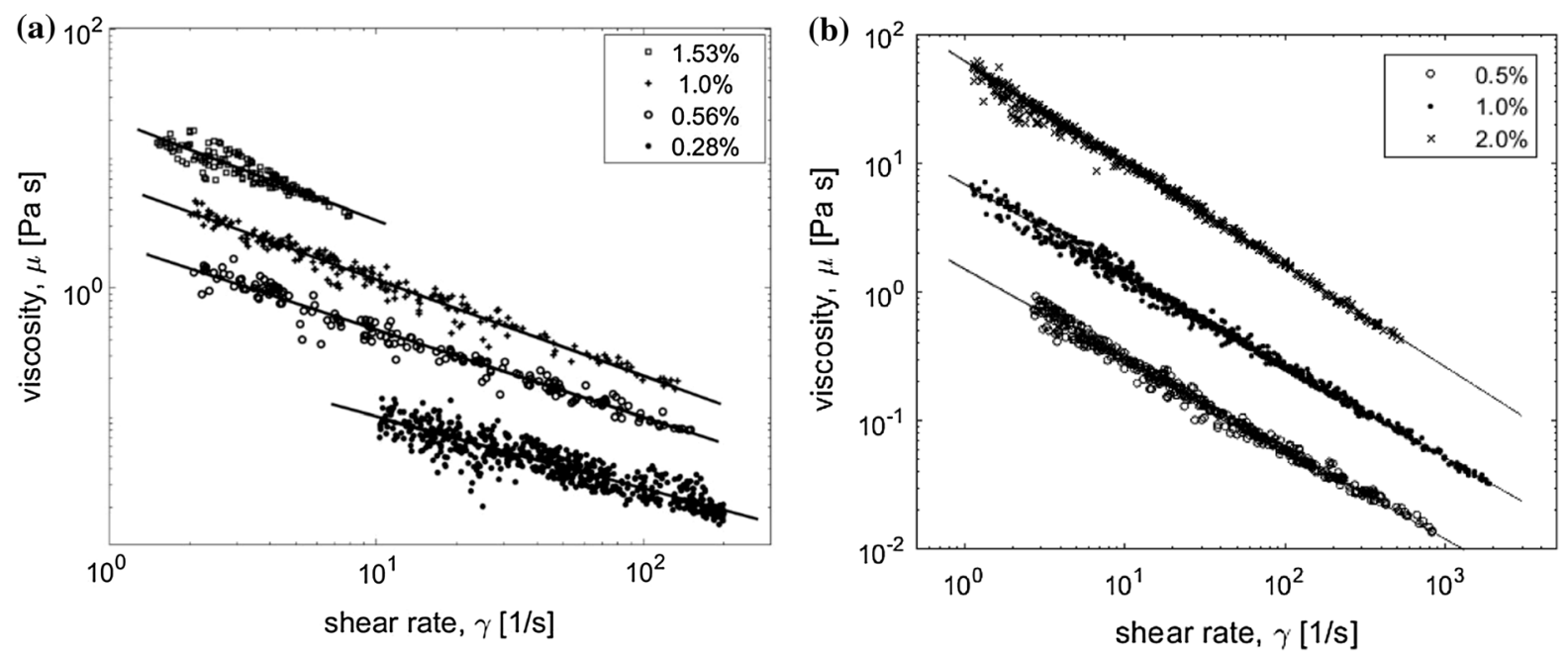

Fig. 8 Viscosity of $\mathbf{a}$ MFC1 and $\mathbf{b}$ MFC2 as a function of shear rate determined with a local derivative Eq. (4)

Table 1 The coefficients of power law Eq. (2) obtained with a local derivative and with velocity profile fitting

\begin{tabular}{llllll}
\hline$c(\%)$ & \multicolumn{2}{l}{ Derivative } & & \multicolumn{2}{l}{ Profile fit } \\
\cline { 6 - 6 } \cline { 5 - 6 } & $K$ & $n$ & & $K$ & $n$ \\
\hline$M F C 1$ & & & & \\
0.17 & 0.10 & 0.51 & & 0.042 & 0.73 \\
0.28 & 0.35 & 0.45 & & 0.27 & 0.51 \\
0.56 & 2.3 & 0.32 & & 2.2 & 0.33 \\
0.80 & 3.8 & 0.30 & & 3.8 & 0.30 \\
1.0 & 6.5 & 0.26 & & 6.3 & 0.27 \\
1.14 & 9.9 & 0.28 & & 11 & 0.26 \\
1.53 & 20 & 0.22 & & 16 & 0.30 \\
$M F C 2$ & & & & 0.31 \\
0.50 & 1.5 & 0.30 & 1.7 & 0.26 \\
1.0 & 6.9 & 0.29 & 9.0 & \\
2.0 & 62 & 0.21 & 59 & \\
\hline
\end{tabular}

Nazari et al. (2016) studied mechanical MFC in the consistency range of 2-7\% and obtained an exponent of 2.3. Equation (12) is thus well in line with the results of earlier studies.

An interesting link to pulp suspension can be found here with the energy dissipation $\varepsilon_{f}$ needed for floc level fluidization of pulps-a phenomenon which is closely related to pulp viscosity. According to Bennington and Kerekes (1996), this quantity scales as $\varepsilon_{f} \propto c^{k}$, with $k=2.5$ for softwood Kraft pulps. The exponent is thus close to what was obtained here for the consistency index of MFC suspensions.

We can see from Fig. 9 that the overall viscous behavior of both MFCs is very similar, although they have clearly different particle size. This is not as surprising as it first seems. Dimensional analysis shows that for low Reynolds number flows of spherical particles, the viscosity of a suspension is a function of only two factors: carrier fluid viscosity and consistency - the particle size does not affect it (Mewis and Wagner 2012). The viscosity of suspensions of elongated particles also depends on the aspect ratio of particles. Two fiber suspensions can thus have very similar viscous behavior even though their particle size differs considerably.

Lasseuguette et al. (2008) and Geng et al. (2017) have shown that below the gel point with a fixed share rate the slope of viscosity versus consistency curve is much shallower. (In Geng et al., e.g., $\mu \sim c^{0.4}$ below the gel point with the shear rate of $100 \mathrm{1} / \mathrm{s}$ ). Obviously the same is the case with the consistency index versus consistency curve. We see from Fig. 9b that all the data points of MFC1 fall on the same power law line. The MFC1 measurements have thus been performed in a consistency range where consistency is at and above the gel point.

Figure 10 shows the consistency index as a function of yield stress. We can see that the relationship between these two quantities is fully linear for MFC1. For MFC2, there are too few points for any real conclusions, but the relationship here also appears to 

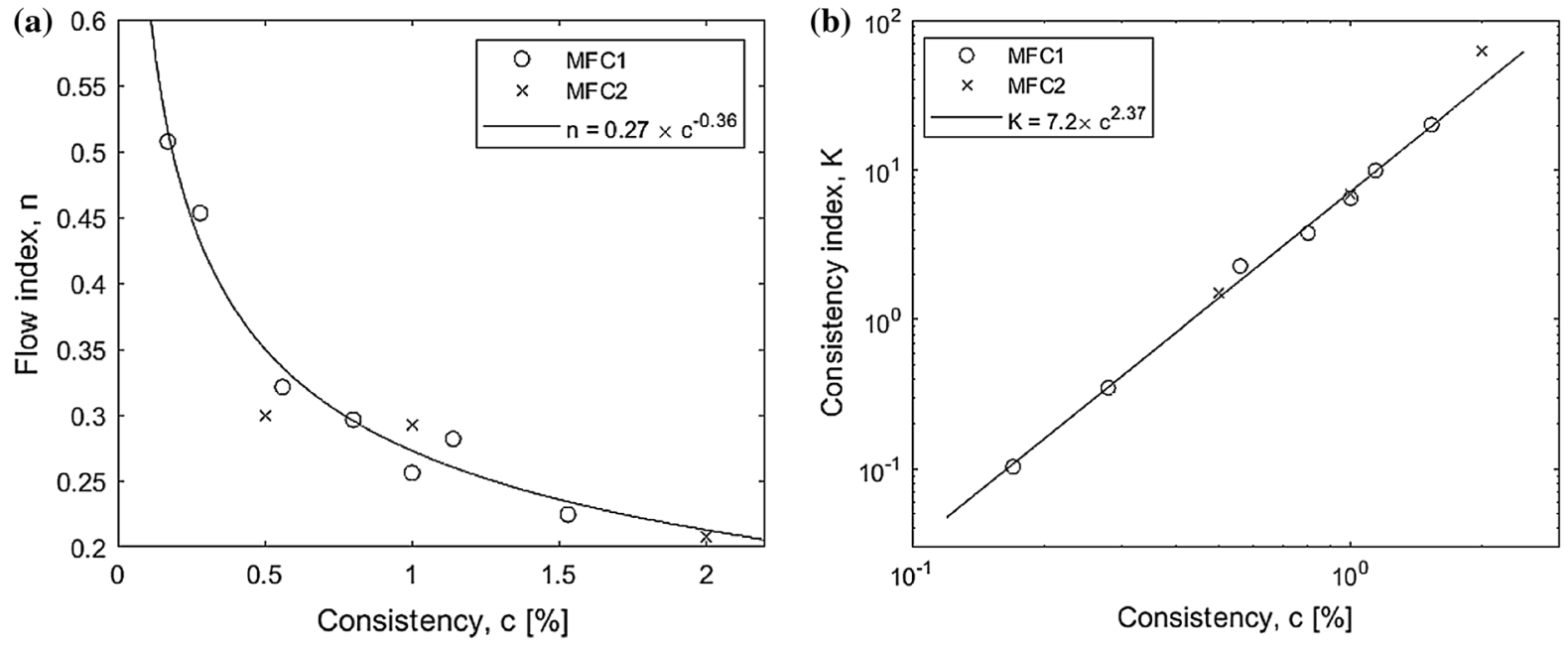

Fig. 9 a Flow indices $n$ for MFC1 and MFC2. The solid line shows a power law fit to all data points. b Consistency indices $K$ for MFC1 and MFC2. The solid line shows a power law fit to MFC1 data points. A power law fit to MFC2 data points gives $K=8.6 \times c^{2.68}$

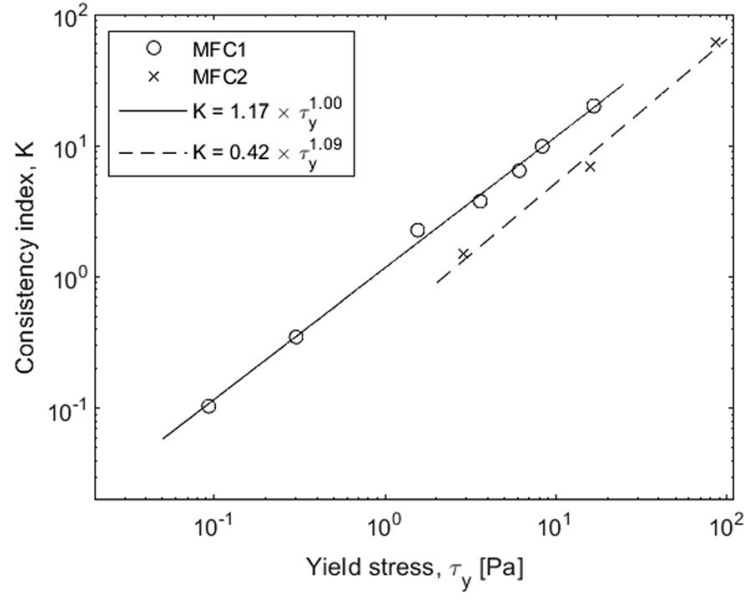

Fig. 10 Consistency index as a function of yield stress for MFC1 and MFC2

be close to linear. We are unaware of earlier studies in which this relationship has been studied experimentally or theoretically. Obviously, both the yield stress and viscosity depend on interactions between the fibrils. Under flow conditions, however, an increase of shear rate tends to enhance both the aggregation and fragmentation of particle clusters and the (gel-like) structure of MFC should be strongly altered (Hubbe et al. 2017). It is thus possible that the observed (linear) behavior is not universal and is limited to certain types of MFC grades.

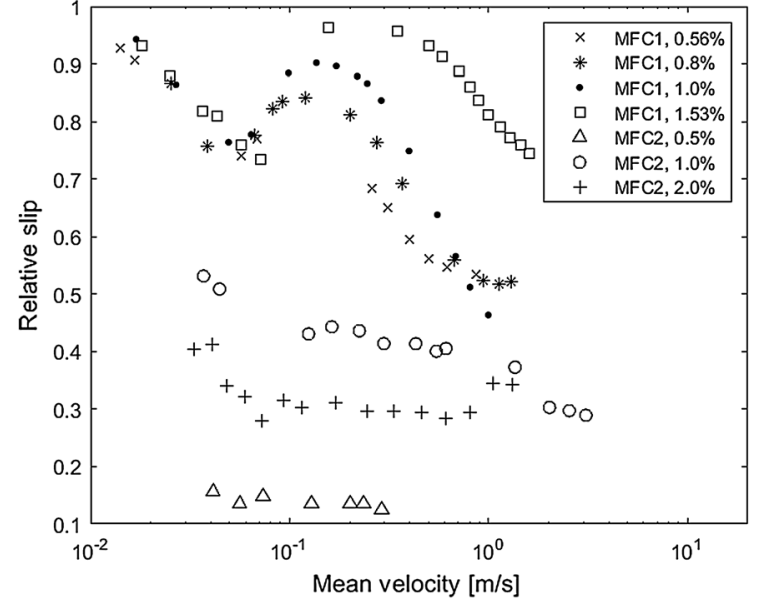

Fig. 11 Relative slip as a function of mean velocity for some consistencies of MFC1 and MFC2 in the vertical pipe. Some data points have been omitted or combined to make the graph easier to read

\section{Wall slip}

Figure 11 shows the relative slip for some consistencies of MFC1 and MFC2 in the vertical pipe as a function of mean velocity. We can see in Fig. 11 that the relative slip is clearly stronger for MFC1: its contribution to flow rate is over $50 \%$ in the measured velocity range. We can also see that for MFC1 the relative slip decreases monotonically until the mean velocity exceeds $0.07 \mathrm{~m} / \mathrm{s}$. At this point, the relative slip increases abruptly, the effect being stronger for 

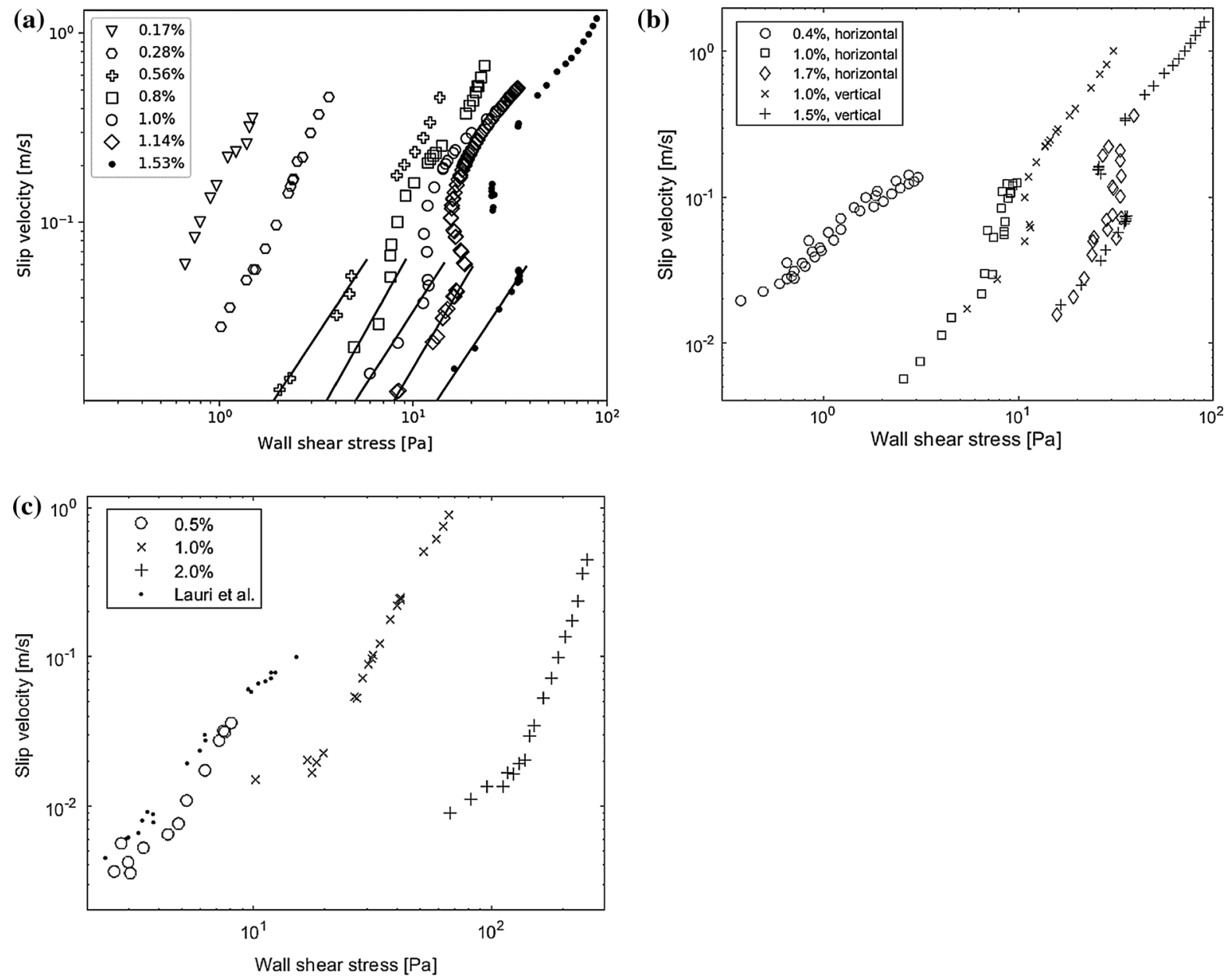

Fig. 12 a Slip velocity as a function of wall shear stress for MFC1 in the vertical pipe. The solid lines are fits of the power law Eq. (13) to the measurement data. b Slip velocity for MFC1 in the horizontal pipe. For comparison, slip velocities for the

higher consistencies. Then the relative slip starts to decrease again. The behavior of MFC2 is very different-here relative slip decreases more or less monotonously with increasing mean velocity and the highest slip is seen with $1.0 \%$ consistency.

We can see from Fig. 11 that the slip behavior of the MFC suspensions can be very complex. Understanding the slip dynamics would require detailed measurements of the dynamics of the depletion layer-e.g. the development of wall consistency profile should be measured in situ. This is a challenging topic of its own, and is outside the scope of this paper. Below we will look at the slip behavior as a function of wall shear stress, as unlike mean velocity, it is a local quantity (Fig. 12).

vertical pipe have been shown for two consistencies. c Slip velocity of MFC2 in the vertical pipe. The results of Lauri et al. (2017) were obtained for $0.5 \%$ MFC2 in a horizontal $8.6 \mathrm{~mm}$ pipe

With many flowing materials, the slip velocity is a function of wall shear stress, and the relationship between the two quantities is a power law (Jäsberg et al. 2015; Cloitre and Bonnecaze 2017)

$v_{s}=S \tau_{w}^{m}$.

This also appears to be the case here, but the parameters of the power law vary with consistency and MFC type. Figure 12a shows the slip velocity of MFC1 as a function of wall shear stress for the horizontal pipe. For consistencies higher than $0.5 \%$, the slip curve can be divided into three sections: With the smallest wall shear rates the slip velocity follows the power law Eq. (13) with $m \approx 1.6$. When wall shear stress reaches approximately two times the yield stress 
(we call this drag reduction threshold stress, $\tau_{D R}$ ), the slip behavior changes. Here slip velocity increases significantly, and there is strong drag reduction of the flow-pressure loss levels or even drops (see Fig. 5a, b). After the drag reduction region, slip velocity once again increases monotonously. Note that the slip velocity at $\tau_{D R}$ is approximately constant, $0.06 \mathrm{~m} / \mathrm{s}$. This indicates that hydrodynamic lift forces might be involved in the development of the wall depletion layer (Jäsberg et al. 2000).

The slip velocity of MFC1 in the horizontal pipe is shown in Fig. 12b. For comparison, slip velocities for $1.0 \%$ and $1.5 \%$ MFC1 in a vertical pipe are also shown. We can see that the slip behavior is rather similar in both pipes, although the pipe diameters are different. This suggests that the wall slip of MFC1 was indeed a function of wall shear stress and independent of the exact details of the flow geometry (see also Fig. $12 \mathrm{c}$ for $0.5 \% \mathrm{MFC} 2$ ).

Figure $12 \mathrm{c}$ shows the slip velocity for MFC2. The results of Lauri et al. (2017) for $0.5 \%$ MFC2 in a horizontal $8.6 \mathrm{~mm}$ pipe are shown for comparison. The slip velocities in Lauri et al. (2017) and in this study are rather similar. The differences might be due to different MFC2 batches, or to experimental uncertainties. Slip velocities can also be calculated from $v_{s}=v-v_{P L}$, where $v$ is the mean velocity in the pipe and

$v_{P L}=\frac{n R^{(n+1) / n}}{3 n+1}\left(-\frac{\nabla P}{K}\right)^{1 / n}$

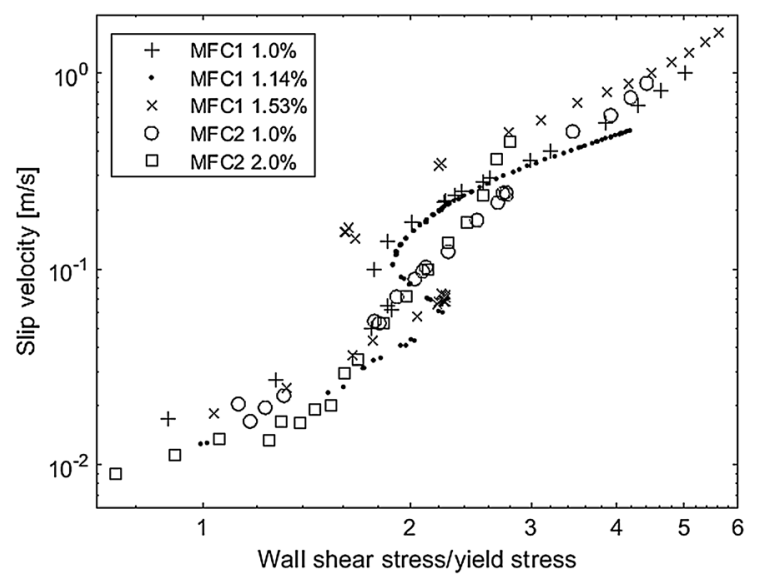

Fig. 13 Slip velocity as a function of wall shear stress scaled with yield stress. All measurements were obtained with the vertical pipe is the theoretical flow rate of a power law fluid in a pipe. With the parameters $K=1.7$ and $n=0.31$, obtained with velocity field fitting (see Table 1) for $0.5 \%$ MFC2, slip velocities are obtained that are close to those obtained by Lauri et al. (2017).

Figure 13 shows the slip velocity for higher consistencies of MFC1 and MFC2 as a function of shear stress, scaled with the yield stress obtained from Eq. (10). We can see that there is a good collapse of data. This is remarkable, as there is an order of magnitude difference in the thickness of the wall depletion layers of MFC1 and MFC2 (Salmela et al. 2013; Lauri et al. 2017). The slip curve appears to consist of three regions: a power law region $\left(\tau_{w} / \tau_{y}<1.5\right)$ where $v_{s}=0.014\left(\tau_{w} / \tau_{y}\right)^{1.5}$, a transition region $\left(1.5<\tau_{w} / \tau_{y}<2.5\right)$, and a power law region $\left(2.5<\tau_{w} / \tau_{y}\right)$ where $v_{s}=0.039\left(\tau_{w} / \tau_{y}\right)^{2.0}$.

\section{Conclusions}

We studied the rheology of two mechanically manufactured MFC suspensions in a pipe rheometer in the consistency range of $0.2-2.0 \%$. Despite having significantly different particle sizes, the viscous behaviors of the suspensions were very similar-the dependences of the flow index and the consistency index on consistency were almost identical. The yield stress of the fine MFC2 was almost threefold higher than for the coarse MFC1, while a $20 \%$ fines fraction of MFC1 measured in Varanasi et al. (2013) gave an almost identical yield stress to MFC1.

Interestingly, the dependence of yield stress and the consistency index on consistency was a power law with an exponent of ca 2.4 for both suspensions. Similar scaling behavior has been seen earlier for some pulp fiber suspensions. For both suspensions there was a linear relationship between the yield stress and consistency index. We have currently no theoretical explanation for this behavior.

Both MFC suspensions had strong slip flow on the pipe walls, and slip contributed up to $95 \%$ of the flow rate. When wall shear stress exceeded two times the yield stress, slip caused drag reduction with consistencies higher than $0.8 \%$. With consistencies higher than $1 \%$, a data collapse was obtained when the slip velocity of both MFC suspensions was presented as a 
function of wall shear stress scaled with the yield stress. This curve consisted of three regions: a power law region for $\tau_{w} / \tau_{y}<1.5$, a transition region that was centered at $\tau_{w} / \tau_{y} \sim 2$, and a power law region for $2.5<\tau_{w} / \tau_{y}$. This result suggests that despite its apparent complexity it might be possible to develop more general models for slip behavior of MFC, at least for moderate consistencies.

The similarities in the shear rheology of the MFC suspensions studied here and in some other studies, and the similar behavior of some pulp fiber suspensions, suggests that the shear rheology of MNFC suspensions might be more universal than has previously been understood. In their basic form (i.e. with low ionic strength and without extra polymers or surface modification), MNFCs might be "just" another group of fibrous materials with a high aspect ratio. Although it is obvious that the MNFC raw material and the production details (mechanical, chemical or enzymatic) may strongly affect the absolute values of the rheological parameters, it is possible that the scaling laws are similar for a wider group of MNFC materials than previously believed. The variability of reported scaling laws of these materials found in the literature might be due not only to real differences in their physical behavior, but also to experimental uncertainties and the general difficulty of measuring their rheological behavior rigorously. The possible wider universality of shear rheology of MNFC materials definitely merits further investigation in the future.

Acknowledgments Open access funding provided by VTT Technical Research Centre of Finland Ltd. This project has received funding from the European Union's Horizon 2020 research and innovation programme under Grant Agreement No. 713475. The work was a part of the Academy of Finland's Flagship Programme under Project No. 318891 (Competence Center for Materials Bioeconomy, FinnCERES). We thank prof. Markku Kataja for OCT data analysis.

\section{Compliance with ethical standards}

Conflict of interest The authors declare no conflict of interest.

Open data https://zenodo.org/record/3413909

Open Access This article is distributed under the terms of the Creative Commons Attribution 4.0 International License (http:// creativecommons.org/licenses/by/4.0/), which permits unrestricted use, distribution, and reproduction in any medium, provided you give appropriate credit to the original author(s) and the source, provide a link to the Creative Commons license, and indicate if changes were made.

\section{References}

Agoda-Tandjawa G, Durand S, Berot S, Blassel C, Gaillard C, Garnier C, Doublier J (2010) Rheological characterization of microfibrillated cellulose suspensions after freezing. Carbohydr Polym 80(3):677-686. https://doi.org/10.1016/ j.carbpol.2009.11.045

Agoda-Tandjawa G, Durand S, Gaillard C, Garnier C, Doublier J (2012) Rheological behaviour and microstructure of microfibrillated cellulose suspensions/low-methoxyl pectin mixed systems. Effect of calcium ions. Carbohydr Polym 87(2):1045-1057. https://doi.org/10.1016/j. carbpol.2011.08.021

Andresen M, Stenius P (2007) Water-in-oil emulsions etabilized by hydrophobized microfibrillated cellulose. J Dispers Sci Technol 28(6):837-844. https://doi.org/10.1080/ 01932690701341827

Bennington C, Kerekes R (1996) Power requirements for pulp suspension fluidization. Tappi J 79(2):253-258

Bounoua S, Lemaire E, Férec J, Ausias G, Kuzhir P (2016) Shear-thinning in concentrated rigid fiber suspensions: aggregation induced by adhesive interactions. J Rheol 60(6):1279-1300. https://doi.org/10.1122/1.4965431

Charani R, Dehghani-Firouzabadi M, Afra E, Shakeri A (2013) Rheological characterization of high concentrated MFC gel from kenaf unbleached pulp. Cellulose 20(2):727-740. https://doi.org/10.1007/s10570-013-9862-1

Cloitre M, Bonnecaze R (2017) A review on wall slip in high solid dispersions. Rheol Acta 56(3):283-305. https://doi. org/10.1007/s00397-017-1002-7

Colson J, Bauer W, Mayr M, Fischer W, Gindl-Altmutter W (2016) Morphology and rheology of cellulose nanofibrils derived from mixtures of pulp fibres and papermaking fines. Cellulose 23(4):2439-2448. https://doi.org/10.1007/ s10570-016-0987-x

Dalpke B, Kerekes R (2005) The influence of pulp properties on the apparent yield stress of flocculated fibre suspensions. J Pulp Pap Sci 31(1):39-43

Delisée C, Lux J, Malvestio J (2010) 3D morphology and permeability of highly porous cellulosic fibrous material. Transp Porous Med 83:623-636

Derakhshandeh B, Kerekes RJ, Hatzikiriakos SG, Bennington CPJ (2011) Rheology of pulp fibre suspensions: a critical review. Chem Eng Sci. https://doi.org/10.1016/j.ces.2011. 04.017

Desmaisons J, Boutonnet E, Rueff M, Dufresne A, Bras J (2017) A new quality index for benchmarking of different cellulose nanofibrils. Carbohydr Polym 174:318-329. https:// doi.org/10.1016/j.carbpol.2017.06.032

Dimic-Misic K, Gane PAC, Paltakari J (2013a) Micro and nanofibrillated cellulose as a rheology modifier additive in CMC-containing pigment-coating formulations. Ind Eng Chem Res 52(45):16066-16083. https://doi.org/10.1021/ ie 4028878

Dimic-Misic K, Puisto A, Gane P, Nieminen K, Alava M, Paltakari J, Maloney T (2013b) The role of MFC/NFC 
swelling in the rheological behavior and dewatering of high consistency furnishes. Cellulose 20(6):2847-2861. https:// doi.org/10.1007/s10570-013-0076-3

Drexler W, Fujimoto J (2008) Optical coherence tomography, technology and applications. Springer, Berlin

Geng L, Naderi A, Mao Y, Zhan C, Sharma P, Peng X, Hsiao B (2017) Rheological properties of jute-based cellulose nanofibers under different ionic conditions. In: Agarwal UP, Atalla RH, Isogai A (eds) Nanocelluloses: their preparation, properties, and applications. American Chemical Society, Washington, DC, pp 113-132

Gourlay K, van der Zwan T, Shourav M, Saddler J (2018) The potential of endoglucanases to rapidly and specifically enhance the rheological properties of micro/nanofibrillated cellulose. Cellulose 25(2):977-986. https://doi.org/10. 1007/s10570-017-1637-7

Haavisto S, Liukkonen J, Jäsberg A, Koponen A, Lille M, Salmela J (2011) Laboratory-scale pipe rheometry: a study of a microfibrillated cellulose suspension. In: Paper conference and trade show 2011, PaperCon 2011, vol 1, pp 357-370

Haavisto S, Salmela J, Jäsberg A, Saarinen T, Karppinen A, Koponen A (2015a) Rheological characterization of microfibrillated cellulose suspension using optical coherence tomography. Tappi J 14(5):291-302

Haavisto S, Salmela J, Koponen A (2015b) Accurate velocity measurements of boundary-layer flows using Doppler optical coherence tomography. Exp Fluids 56(5):96. https://doi.org/10.1007/s00348-015-1962-2

Haavisto S, Cardona MJ, Salmela J, Powell RL, McCarthy MJ, Kataja M, Koponen AI (2017) Experimental investigation of the flow dynamics and rheology of complex fluids in pipe flow by hybrid multi-scale velocimetry. Exp Fluids 58(11):1-13. https://doi.org/10.1007/s00348-017-2440-9

Hoeng F, Denneulin A, Reverdy-Bruas N, Krosnicki G, Bras J (2017) Rheology of cellulose nanofibrils/silver nanowires suspension for the production of transparent and conductive electrodes by screen printing. Appl Surf Sci 394:160-168. https://doi.org/10.1016/j.apsusc.2016.10. 073

Hubbe M, Tayeb P, Joyce M, Tyagi P, Kehoe M, Dimic-Misic K, Pal L (2017) Rheology of nanocellulose-rich aqueous suspensions: a review. BioResources 12(4):9556-9661

Iotti M, Gregersen O, Moe S, Lenes M (2011) Rheological studies of microfibrillar cellulose water dispersions. J Polym Environ 19(1):137-145

Isogai A (2013) Wood nanocelluloses: fundamentals and applications as new bio-based nanomaterials. J Wood Sci 59(6):449-459. https://doi.org/10.1007/s10086-013-1365$\mathrm{z}$

Jäsberg A (2007) Flow behaviour of fibre suspensions in straight pipes: new experimental techniques and multiphase modeling. University of Jyväskylä, Jyväskylä

Jäsberg A, Koponen A, Kataja M, Timonen J (2000) Hydrodynamical forces acting on particles in a two-dimensional flow near a solid wall. Comput Phys Commun 129(1):196-206

Jäsberg A, Selenius P, Koponen A (2015) Experimental results on the flow rheology of fiber-laden aqueous foams. Colloids Surf A 473:147-155. https://doi.org/10.1016/j. colsurfa.2014.11.041
Jowkarderis L, van de Ven T (2014) Intrinsic viscosity of aqueous suspensions of cellulose nanofibrils. Cellulose 21(4):2511-2517. https://doi.org/10.1007/s10570-0140292-5

Jowkarderis L, van de Ven T (2015) Rheology of semi-dilute suspensions of carboxylated cellulose nanofibrils. Carbohydr Polym 123:416-423. https://doi.org/10.1016/j. carbpol.2015.01.067

Karppinen A, Vesterinen A, Saarinen T, Pietikäinen P, Seppälä J (2011) Effect of cationic polymethacrylates on the rheology and flocculation of microfibrillated cellulose. Cellulose 18(6):1381-1390. https://doi.org/10.1007/s10570011-9597-9

Karppinen A, Saarinen T, Salmela J, Laukkanen A, Nuopponen M, Seppälä J (2012) Flocculation of microfibrillated cellulose in shear flow. Cellulose 19(6):1807-1819. https:// doi.org/10.1007/s10570-012-9766-5

Kataja M, Haavisto S, Salmela J, Lehto R, Koponen A (2017) Characterization of micro-fibrillated cellulose fiber suspension flow using multi scale velocity profile measurements. Nord Pulp Pap Res J 32(3):473-482. https://doi.org/ 10.3183/NPPRJ-2017-32-03-p473-482

Kinnunen-Raudaskoski K, Lehmonen J, Beletski N, Jetsu P, Hjelt T (2013) Benefits of foam forming technology and its applicability in high NFC addition structures. In: Proceedings of the 15th pulp and paper fundamental research symposium, Cambridge, Oxford

Klemm D, Kramer F, Moritz S, Lindström T, Ankerfors M, Gray D, Dorris A (2011) Nanocelluloses: a new family of naturebased materials. Angew Chem Int Ed 50(24):5438-5466. https://doi.org/10.1002/anie.201001273

Kotze R, Wiklund J, Haldenwang R (2013) Optimisation of Pulsed Ultrasonic Velocimetry system and transducer technology for industrial applications. Ultrasonics 53:459-469. https://doi.org/10.1016/j.ultras.2012.08.014

Kumar V, Ottesen V, Syverud K, Gregersen Ø, Toivakka M (2017) Coatability of cellulose nanofibril suspensions: role of rheology and water retention. BioResources 12(4):7656-7679. https://doi.org/10.15376/biores.12.4. 7656-7679

Lasseuguette E, Roux D, Nishiyama Y (2008) Rheological properties of microfibrillar suspension of TEMPO-oxidized pulp. Cellulose 15(3):425-433. https://doi.org/10. 1007/s10570-007-9184-2

Lauri J, Koponen A, Haavisto S, Czajkowski J, Fabritius T (2017) Analysis of rheology and wall depletion of microfibrillated cellulose suspension using optical coherence tomography. Cellulose 24:4715-4728. https://doi.org/ 10.1007/s10570-017-1493-5

Li MC, Wu Q, Song K, Qing Y, Wu Y (2015) Cellulose nanoparticles as modifiers for rheology and fluid loss in bentonite water-based fluids. ACS Appl Mater Interfaces 7(8):5009-5016. https://doi.org/10.1021/acsami.5b00498

Lowys M, Desbrières J, Rinaudo M (2001) Rheological characterization of cellulosic microfibril suspensions. Role of polymeric additives. Food Hydrocoll 15(1):25-32. https:// doi.org/10.1016/S0268-005X(00)00046-1

Martoia F, Perge C, Dumont P, Orgeas L, Fardin M, Manneville S, Belgacem M (2015) Heterogeneous flow kinematics of cellulose nanofibril suspensions under shear. Soft Matter 11(24):4742-4755. https://doi.org/10.1039/c5sm00530b 
Mewis J, Wagner N (2012) Colloidal suspension rheology. Cambridge University Press, New York

Mohtaschemi M, Dimic-Misic K, Puisto A, Korhonen M, Thaddeus M, Paltakari J, Alava M (2014a) Rheological characterization of fibrillated cellulose suspensions via bucket vane viscometer. Cellulose 21:1305-1312

Mohtaschemi M, Sorvari A, Puisto A, Nuopponen M, Seppälä J, Alava M (2014b) The vane method and kinetic modeling: shear rheology of nanofibrillated cellulose suspensions. Cellulose 21(6):3913-3925. https://doi.org/10.1007/ s10570-014-0409-x

Moon R, Schueneman G, Simonsen J (2016) Overview of cellulose nanomaterials, their capabilities and applications. J Miner Met Mater Soc 68(9):2383-2394. https://doi.org/ 10.1007/s11837-016-2018-7

Mosse WKJ, Boger DV, Garnier G (2012) Avoiding slip in pulp suspension rheometry. J Rheol 56(6):1517-1533. https:// doi.org/10.1122/1.4752193

Naderi A (2017) Nanofibrillated cellulose: properties investigated. Cellulose 24:1933-1945

Naderi A, Lindström T (2014) Carboxymethylated nanofibrillated cellulose: effect of monovalent electrolytes on the rheological properties. Cellulose 24:3507-3514. https:// doi.org/10.1007/s10570-014-0394-0

Nazari B, Kumar V, Bousfield D, Toivakka M (2016) Rheology of cellulose nanofibers suspensions: boundary driven flow. J Rheol 60(6):1151-1159. https://doi.org/10.1122/1. 4960336

Nechyporchuk O, Belgacem MN, Pignon F (2014) Rheological properties of micro-/nanofibrillated cellulose suspensions: wall-slip and shear banding phenomena. Carbohydr Polym 112:432-439. https://doi.org/10.1016/j.carbpol.2014.05. 092

Nechyporchuk O, Belgacem M, Pignon F (2016) Current progress in rheology of cellulose nanofibril suspensions. Biomacromolecules 17(7):2311-2320. https://doi.org/10. 1021/acs.biomac.6b00668

Pääkkönen T, Dimic-Misic K, Orelma H, Pönni R, Vuorinen T, Maloney T (2016) Effect of xylan in hardwood pulp on the reaction rate of TEMPO-mediated oxidation and the rheology of the final nanofibrillated cellulose gel. Cellulose 23(1):277-293. https://doi.org/10.1007/s10570-015-08247

Petrich M, Koch D, Cohen C (2000) Experimental determination of the stress-microstructure relationship in semi-concentrated fiber suspensions. J NonNewton Fluid Mech 95:101-133. https://doi.org/10.1016/S03770257(00)00172-5

Powell R (2008) Experimental techniques for multiphase flows. Phys Fluids 20(4):040605. https://doi.org/10.1063/1. 2911023

Raj P, Mayahi A, Lahtinen P, Varanasi S, Garnier G, Martin D, Batchelor W (2016) Gel point as a measure of cellulose nanofibre quality and feedstock development with mechanical energy. Cellulose 23(5):3051-3064. https:// doi.org/10.1007/s10570-016-1039-2

Saarikoski E, Saarinen T, Salmela J, Seppälä J (2012) Flocculated flow of microfibrillated cellulose water suspensions: an imaging approach for characterisation of rheological behaviour. Cellulose 19(3):647-659. https://doi.org/10. 1007/s10570-012-9661-0
Saarikoski E, Rissanen M, Seppälä J (2015) Effect of rheological properties of dissolved cellulose/microfibrillated cellulose blend suspensions on film forming. Carbohydr Polym 119:62-70. https://doi.org/10.1016/j.carbpol.2014. 11.033

Saarinen T, Haavisto S, Sorvari A, Salmela J, Seppälä J (2014) The effect of wall depletion on the rheology of microfibrillated cellulose water suspensions by optical coherence tomography. Cellulose 21(3):1261-1275. https://doi.org/ 10.1007/s10570-014-0187-5

Salmela J, Haavisto S, Koponen A, Jäsberg A, Kataja M (2013) Rheological characterization of micro-fibrillated cellulose fibre suspension using multi scale velocity profile measurements. In: 15th Fundamental research symposium, pp 495-509

Samyn P, Taheri H (2016) Rheology of fibrillated cellulose suspensions after surface modification by organic nanoparticle deposits. J Mater Sci 51(21):9830-9848. https://doi.org/10.1007/s10853-016-0216-X

Schenker M, Schoelkopf J, Gane P, Mangin P (2018) Influence of shear rheometer measurement systems on the rheological properties of microfibrillated cellulose (MFC) suspensions. Cellulose 25:961-976

Shao Y, Chaussy D, Grosseau P, Beneventi D (2015) Use of microfibrillated cellulose/lignosulfonate blends as carbon precursors: impact of hydrogel rheology on $3 \mathrm{D}$ printing. Ind Eng Chem Res 54(43):10575-10582. https://doi.org/ 10.1021/acs.iecr.5b02763

Swerin A, Odberg L, Lindström T (1990) Deswelling of hardwood kraft pulp fibers by cationic polymers. Nord Pulp Pap Res J 5:188-196. https://doi.org/10.3183/npprj-1990-0504-p188-196

Takeda Y (ed) (2012) Ultrasonic Doppler velocity profiler for fluid flow. Springer Japan, Tokyo

Tatsumi D, Ishioka S, Matsumoto T (2002) Effect of fiber concentration and axial ratio on the rheological properties of cellulose fiber suspensions. Nihon Reoroji Gakkaishi 30(1):27-32. https://doi.org/10.1678/rheology.30.27

Vadodaria S, Onyianta A, Sun D (2018) High-shear rate rheometry of micro-nanofibrillated cellulose (CMF/CNF) suspensions using rotational rheometer. Cellulose 4:5535-5552. https://doi.org/10.1007/s10570-018-1963-4

Varanasi S, He R, Batchelor W (2013) Estimation of cellulose nanofibre aspect ratio from measurements of fibre suspension gel point. Cellulose 20(4):1885-1896. https://doi. org/10.1007/s10570-013-9972-9

Winuprasith T, Suphantharika M (2013) Microfibrillated cellulose from mangosteen (Garcinia mangostana L.) rind: preparation, characterization, and evaluation as an emulsion stabilizer. Food Hydrocoll 32(2):383-394. https://doi. org/10.1016/j.foodhyd.2013.01.023

Zhang J, Song H, Lin L, Zhuang J, Pang C, Liu S (2012) Microfibrillated cellulose from bamboo pulp and its properties. Biomass Bioenergy 39:78-83. https://doi.org/10. 1016/j.biombioe.2010.06.013

Publisher's Note Springer Nature remains neutral with regard to jurisdictional claims in published maps and institutional affiliations. 